\title{
MAGNETISM IN FeRh ALLOYS
}

\author{
M. Pugacheva and A. Jezierski \\ Institute of Molecular Physics, Polish Academy of Sciences \\ Smoluchowskiego 17/19, 60-179 Poznań, Poland
}

\begin{abstract}
We study the electronic and magnetic properties of FeRh ordered alloys. The electronic structure is calculated by the tight-binding linear muffin-tin orbital method. We observe the decreasing magnetic moment with increase of the ratio $c / a$ during the structural phase transformation from $\mathrm{CsCl}$ to $\mathrm{CuAu}$ type structure.

PACS numbers: 71.25 Pi, 75.10.Lp
\end{abstract}

\section{Introduction}

The magnetic properties of $\mathrm{Fe}_{1-c} \mathrm{Rh}_{c}$ ordered and disordered alloys were studied in the last years [1-8]. Recently, Miyajima and Yuasa [2] have found that the fcc FeRh alloy was paramagnetic and stable at the room temperature. The change of bcc structure to a mixture phase composed of fcc and bct phases was performed by an application of uniaxial pressure of $2.5 \times 10^{4} \mathrm{~kg} / \mathrm{cm}^{2}$ to the bcc FeRh alloy. The experimental lattice constant for bcc FeRh alloy was $a=2.981 \AA$, and for the disordered fcc FeRh $a=3.731 \AA$. In the bct FeRh Miyajima and Yuasa [2] found $a=2.81 \AA$ and $c=3.38 \AA$ (c/ $a=1.203)$. In this paper we study the change of the electronic structure and magnetic moment in FeRh ordered alloy during the structural transformation from the $\mathrm{CsCl}$ to $\mathrm{CuAu}$ type structure. The method of calculations is presented in Sec. 2 and the main results are discussed in Sec. 3. Finally we give some conclusions.

\section{Method of calculations}

The electronic structure and magnetic moment were calculated by the tight binding linear muffin-tin orbital (TB LMTO) method and the local spin density approximation (LSD) [9]. In the self-consistent band structure calculations we used the von Barth-Hedin [10] parametrization for the local exchange correlation potential. The values of the Wigner-Seitz radii were estimated from the minimum of the total energy. We find $S=2.81$ a.u. in FeRh CsCl-type alloy. The main aim of this paper is an examination of the dependence of magnetic moment on ratio $c / a$ during the structural transformation from $\mathrm{CsCl}$ to $\mathrm{CuAu}$ structure. We assume that the volume of the unit cell is constant. The lattice parameter $a$ and the ratio $c / a$ are changed in such a way to give the same volume of unit cell. 


\section{Results}

We computed the band structure and magnetic moment in FeRh alloy for the different values of $c / a(1<c / a<1.41)$. In Fig. 1 we present the dependence of the total $(\mathrm{FeRh})$ and local $(\mathrm{Fe}, \mathrm{Rh})$ magnetic moment on the ratio $c / a$. In the region $1.224<c / a<1.226$ we find the structural phase transition from

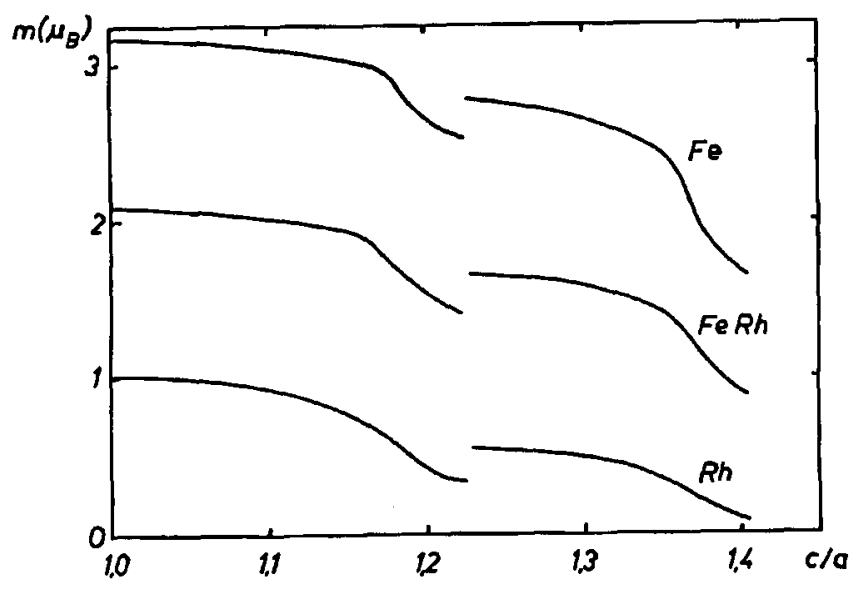

Fig. 1. The $\mathrm{n} \mathbf{n}$ e of the total and local magnetic moment in FeRh on the ratio $c / a$.

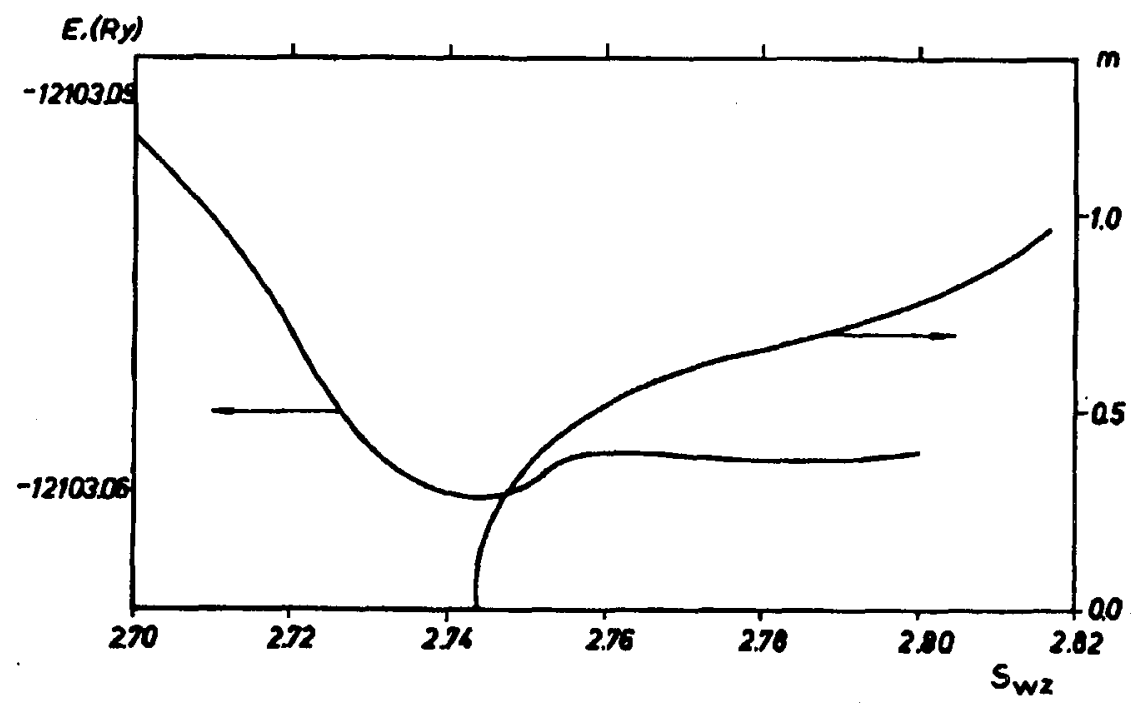

Fig. 2. The plot of total magnetic moment and the total energy versus the Wigner-Seitz radius. 
bct to fct structure. The magnetic moment decreases with increase $c / a$, and for $c / a=1.41$ the total magnetic moment for $\mathrm{FeRh}(\mathrm{CuAu})$ goes to $0.87 \mu_{\mathrm{B}}$. Then we examine the energetic stability of $\mathrm{FeRh}(\mathrm{CuAu})$. In Fig. 2 we present the change of the total magnetic moment and the total energy versus the Wigner-Seitz radius. The total magnetic moment goes to zero for $S=2.757$ a.u. For this value of $S$ the system has the minimum of the total energy. The results obtained in this paper are in good agreement with the TB LMTO coherent potential approximation (CPA) results obtained by Jezierski [6]. Recently Jezierski has shown [6] that the fcc disordered FeRh alloys do not satisfy the Stoner criterion. The similar structural transition was observed by Krasko and Olson during the bcc-fcc lattice deformation in iron [11].

We thank Prof. J.A. Morkowski and Dr A. Szajek for useful discussion. The self-consistent band structure calculations were performed by using TB LMTO programs written by M. van Schilfgaarde et al. from MPI in Stuttgart. This work was supported by the project No. 209959101 of the Committee for Scientific Research.

\section{References}

[1] L.Y. Chen, D.W. Lynch, Phys. Rev. B 37, 10503 (1988).

[2] H. Miyajima, S. Yuasa, J. Magn. Magn. Mater. 104-107, 2025 (1992).

[3] L. Vinokurova, E. Kulatov, A. Vlasov, M. Pardavi-Horvath, J. Phys. Coll. C8, Suppl. 12, C8-99 (1988).

[4] A. Szajek, J.A. Morkowski, J. Magn. Magn. Mater. 115, 171 (1992).

[5] A. Szajek, J.A. Morkowski, Physica, in press.

[6] A. Jezierski, Solid State Commun. 86, 685 (1993).

[7] V.L. Moruzzi, P.M. Marcus, Solid State Commun. 83, 735 (1992).

[8] V.L. Moruzzi, P.M. Marcus, Phys. Rev. B 46, 2864 (1992).

[9] O.K. Andersen, O. Jepsen, M. Sob, in: Electronic Band Structures and its Applications, Springer Verlag Lectures Notes in Physics, Vol. 283, Ed. M. Yussouff, Springer, Berlin 1987, p. 1.

[10] U. von Barth, L. Hedin, J. Phys. C 5, 1629 (1972).

[11] G.L. Krasko, G.B. Olson, Phys. Rev.B 40, 11536 (1989). 